\title{
Knowledge and attitude regarding reproductive and sexual health among school teachers: a descriptive survey
}

\author{
${ }^{1}$ Maharishi Markandeshwar College of Nursing Maharishi Markandeshwar (Deemed to be University), Department of Obstet- \\ rics and Cynaecology, Mullana 133207, Haryana, India \\ ${ }^{2}$ Department of Obstetrics and Gynaecology, Maharishi Markandeshwar College of Nursing, Maharishi \\ Markandeshwar (Deemed to be University), Mullana 133207, Haryana, India, Phone: +91-8059930267, E-mail: \\ poonamsheoran@mmumullana.org \\ ${ }^{3}$ Faculty of Nursing, Maharishi Markandeshwar (Deemed to be University), Mullana 133207, Haryana, India
}

\begin{abstract}
:
Background: Adolescence is a time of evolving from the immaturity of childhood into the maturity of adulthood. This is a stage which requires extra care and protection. Physiologically and physically, adolescents go through a series of transformations as they grow and develop.

Method: A descriptive study design was adopted with 70 teachers selected using a convenience sampling technique from five different government schools of the Ambala District, Haryana. Knowledge and attitude were assessed using a structured knowledge questionnaire and a 5-point Likert scale, respectively. The reliability coefficient of the structured knowledge questionnaire was found to be 0.67 by the Kuder-Richardson method and for the attitude scale, it was found to be 0.7 by Cronbach's $\alpha$.

Results: More than one third (37.1\%) of the teachers were between 31 and 35 years of age. Out of 70 the majority $(75.7 \%)$ of the teachers were female. More than half $(62.9 \%)$ of the teachers had an average level of knowledge and the majority (71.4\%) of the teachers had moderately favorable attitudes regarding reproduction and sexual health. Furthermore, teachers had more knowledge about the reproductive organs and the concept of puberty followed by the concept of reproductive and sexual health and had the least knowledge in the area of contraception.

Conclusion: This study highlights the need for special training and awareness programs regarding reproduction and sexual health among school teachers.
\end{abstract}

Keywords: adolescent, attitude, knowledge, reproduction and sexual health, teachers

DOI: 10.1515/ijamh-2019-0107

Received: May 12, 2019; Accepted: June 6, 2019

\section{Introduction}

Adolescence is a period of transition from childhood to adulthood which include a multiple changes such as physical, intellectual, personality and social developmental changes. Adolescence is the time period from puberty to adulthood and may be roughly divided into three stages: early adolescence (11-14 years); middle adolescence (15-17 years) and late adolescence (18-21 years). Furthermore, as regards physiological growth, seven key intellectual, psychological and social developmental tasks are included in these years of life. The foundational motive of these tasks is to form one's own identity and to prepare for adulthood [1].

Physical changes in adolescents are perhaps the most noted signs that a child has entered adolescence. Puberty is the period in which an individual becomes capable of sexual reproduction. Moreover, puberty is used as a cumulative term to refer to all the physical changes that develop in the young girl or boy as they pass from childhood to adolescence [2]. The adolescent period is one of life's most difficult periods. This is the time when an individual enters the world of responsibilities and experiments with them. Global data has highlighted the burning need to focus on adolescents. The leading causes of death among young adolescents are complicated pregnancies and childbirths and unsafe abortions. Adolescents have the highest rate of unmet need for contraception, they are at high risk for HIV infections with $40 \%$ of newly diagnosed HIV infections occur in the 15-24 year old age group [3]. 
This is a stage which requires extra care and protection. Physiologically and physically, adolescents go through a series of transformations while they grow and develop. The most dramatic changes in puberty involve sexuality. Because of the development of primary sexual characteristics, adolescents become capable of sexual reproduction. Sex is a very subtle subject that is still considered a taboo in Indian culture and is a topic that is not discussed openly. Thus, before formally introducing the topic of sex education, it is extremely important to know the perceptions of people, teachers and school children regarding sexual and reproductive health education to avoid any adverse effects [4].

Data from studies show that about $12.3 \%$ of parents and $36.0 \%$ of teachers feel comfortable in imparting knowledge about reproductive and sexual health, whereas $65.2 \%$ of parents have never discussed such topics with their adolescents, and $21.3 \%$ of teachers and $7.7 \%$ of parents think that adolescents may misuse this type of information [5]. The children of this era are more advanced for their age and are much more intelligent, curious and aware towards every little change happening in the world. They want to explore everything and want to know every little fact, so; when parents or elders try to conceal any secret from them they then try every approach to know that thing. Data from the study revealed that the majority of the parents $(70.67 \%)$ and teachers $(75 \%)$ were against the introduction of sex education. Furthermore, results revealed that most of the parents $(75 \%)$ believed that sex education is against their religion, on the other hand, only $(25 \%)$ of teachers believed this [6].

The importance of delivering sex education in a timely manner to adolescents is emphasized by the current statistical data which shows that almost one in every sixth person on the globe is an adolescent. Worldwide they comprise 1.2 billion of worlds' population. India has the highest number of adolescents, $21 \%$ (about 243 million). These numbers indicate the importance of specifically addressing the healthcare needs of this larger demographic group in India [7].

While reproductive and sexual health is such an important issue in one's life, parents, elders and teachers in India hardly play any compelling role in providing adequate information and knowledge. Also, those who seek guidance from parents may not be satisfied as parents are not able to give satisfactory answers so they may avoid these discussions with them. Some parents even find reproductive and sexual health education against their religious beliefs and avoid these topics. In a study it has been found that $154(15.4 \%)$ of the respondents opposed the introduction of sex education because they believed that it would corrupt the students (4\%), it could lead to sexual experimentation $(4 \%)$ and that it should be the responsibility of the parents at home to teach sex education (5.6\%) [8]. By providing proper guidance and education to the adolescents, complications such as STIs (sexually transmitted diseases) and RTIs (reproductive tract infections), etc. can be easily prevented [9]. Moreover improved knowledge and favorable attitudes will also help in decreasing the morbidity and mortality rates in India which will ensure healthy lives and promote well-being for all at all ages and would also help at a global level by achieving millennium development goals (MDG) 3 (to promote gender equality and empower women), MDG 4 (to reduce child mortality), MDG 5 (to improve maternal health) and MDG 6 (to combat HIV / AIDS, malaria and other diseases) [10] with this background, the study was directed to assess the knowledge and attitude of teachers teaching in different schools of the Ambala District, Haryana.

\section{Materials and methods}

\section{Study design}

This quantitative study was based on descriptive survey design to test the participants.

\section{Design and settings}

The study was conducted in five government schools of Barara and Saha villages of the Ambala District, Haryana, selected using a convenience sampling technique. Data was collected between October 2018 to November 2018 after obtaining clearance from the institutional Ethical Committee of Maharishi Markandeshwar (Deemed to be) University Mullana, Ambala, Haryana.

\section{Setting and sample}

The study participants selected by a convenience sampling technique comprised 70 teachers from five government schools (selected by convenience sampling) of Barara and Saha villages of Ambala District, Haryana. 
Inclusion criteria consisted of teachers who were willing to participate and teachers who were not available at the time of data collection were excluded from the study.

\section{Ethical consideration}

This study was affirmed by the institutional Ethical Committee of Maharishi Markandeshwar (Deemed to be) University Mullana, Ambala, Haryana. Written informed consent was also obtained from all the participants before starting the study.

\section{Tools}

Knowledge and attitude were assessed by using a structured knowledge questionnaire and attitude scale (5point Likert scale). Both tools were validated by nine experts in the various nursing fields. The reliability of the tools was checked. The reliability coefficient of the structured knowledge questionnaire was found to be 0.67 by the Kuder-Richardson- 20 formula and for attitude it was found to be 0.7 by Cronbach's $\alpha$. A structured knowledge questionnaire containing 45 multiple choice questions was used with areas like reproductive and sexual development, pubertal changes during adolescence, concept and importance of reproductive health and prevention of RTIs/STIs. A 5-point Likert scale ranging from strongly agree to strongly disagree containing 30 statements was used, out of which 15 were positive statements and 15 were negative. The maximum score was 150 and the minimum score was 30.

\section{Data collection/procedure}

After obtaining the formal approval from the block education officers ( a government officer who is responsible for dealing with secondry, elementary and mass education of students in his/her block area.) of Barara and Saha villages of the Ambala District, Haryana, a test lasting $55 \mathrm{~min}$ was conducted to assess knowledge and attitude regarding reproductive and sexual health.

\section{Data analysis}

Data were entered into Microsoft Excel 2007 and analyzed using SPSS 20 (IBM Corp. version 20.0. Armonk, NY). Categorical data are presented as mean [standard deviation (SD)] or median based on the distribution of data. Statistical analysis was performed by using descriptive analysis.. A p-value of 0.05 was considered significant.

\section{Results}

Data were analyzed by using SPSS-20. A total of 70 teachers completed the study. In the present study more than one third (37.1\%) of the teachers were between the ages of 31-35 years old. Out of 70 the majority $(77.1 \%)$ of the teachers were female. More than a quarter $(27.1 \%)$ of the teachers had Master's in arts degrees. More than one third $(38.6 \%)$ of the teachers had teaching experience of $\geq 7$ years. The majority $(85.7 \%)$ of the teachers did not teach about reproductive and sexual health. The majority $(85.7 \%)$ of the teachers did not have any experience in teaching about reproductive and sexual health. The majority $(98.6 \%)$ of the teachers did not have any specific training in teaching about reproductive and sexual health. The majority $(88.6 \%)$ of the teachers felt that it is necessary to teach about reproductive and sexual health.

\section{Frequency and percentage of level of knowledge score regarding reproductive and sexual health among teachers}

Data shows (Figure 1) the frequency and percentage of the level of knowledge score regarding reproductive and sexual health among teachers where more than half (62.9\%) of the teachers had an average level of knowledge regarding reproductive and sexual health followed by $(22.9 \%)$ had below average knowledge and least $(1.4 \%)$ of teachers had very good knowledge regarding reproductive and sexual health. 


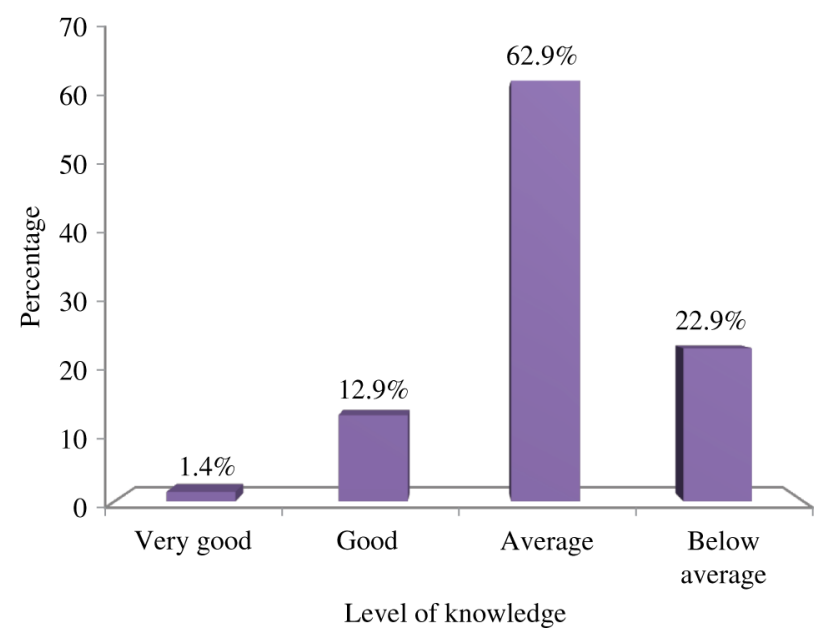

Figure 1: Bar graph showing percentage distribution level of knowledge regarding reproductive and sexual health among teachers.

\section{Range, mean, median and SD of knowledge score regarding reproductive and sexual health among teachers}

The data presented in Table 1 depicts the range, mean, median and SD of knowledge score regarding reproductive and sexual health among teachers. The mean of the knowledge score was 24.84 with a median of 25 and the SD was 3.79.

Table 1: Range, mean, median and SD of knowledge score regarding reproductive and sexual health among teachers.

\begin{tabular}{lrrrr}
\hline & & & $\mathbf{n = 7 0}$ \\
\hline Score & Range & Mean & Median & SD \\
\hline Knowledge & $15-36$ & 24.84 & 25.00 & 3.79 \\
\hline
\end{tabular}

Maximum score - 45; minimum score -0 . SD, standard deviation.

\section{Area-wise mean, mean percentage and SD of knowledge score regarding reproductive and sexual health among teachers}

The data presented in Table 2 depicts the area-wise mean, mean percentage and SD of knowledge score regarding reproductive and sexual health among teachers. The mean score for knowledge about reproductive organs and concept of puberty, concept of reproductive and sexual health, contraception and RTIs and STIs was 10.84, 8.40, 1.76 and 3.84, respectively, which further infer that teachers had more knowledge about reproductive organs and concept of puberty followed by the concept of reproductive and sexual health and least knowledge about contraception.

Table 2: Area wise mean, mean percentage and SD of knowledge score regarding reproductive and sexual health among teachers.

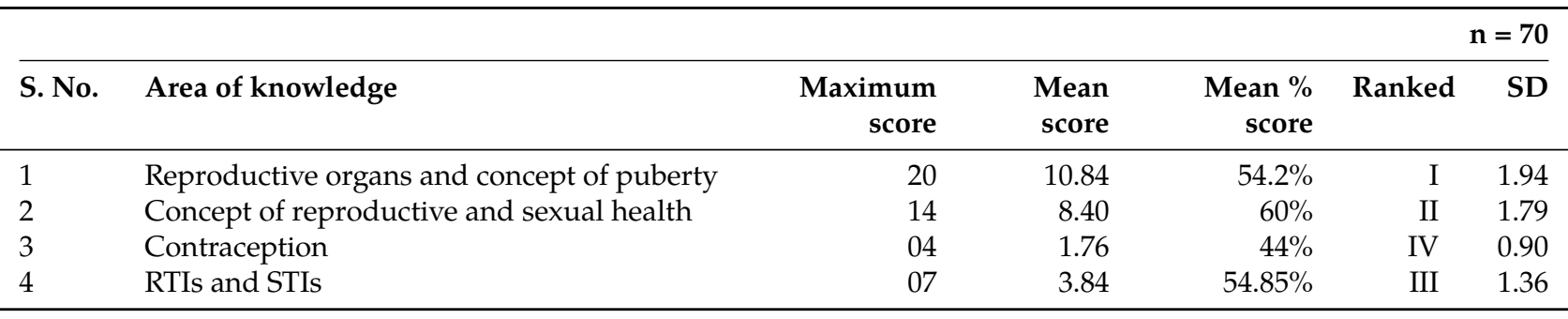


RTIs, reproductive tract infections; STIs, sexually transmitted infections; SD, standard deviation.

\section{Frequency and percentage of level of attitude score regarding reproductive and sexual health among teachers}

Data shows (Figure 2) the frequency and percentage of the level of attitude score regarding reproductive and sexual health among teachers and it found that the majority $(71.4 \%)$ of the teachers had a moderately favorable attitude followed by $(28.6 \%)$ with unfavorable attitude regarding reproductive and sexual health.

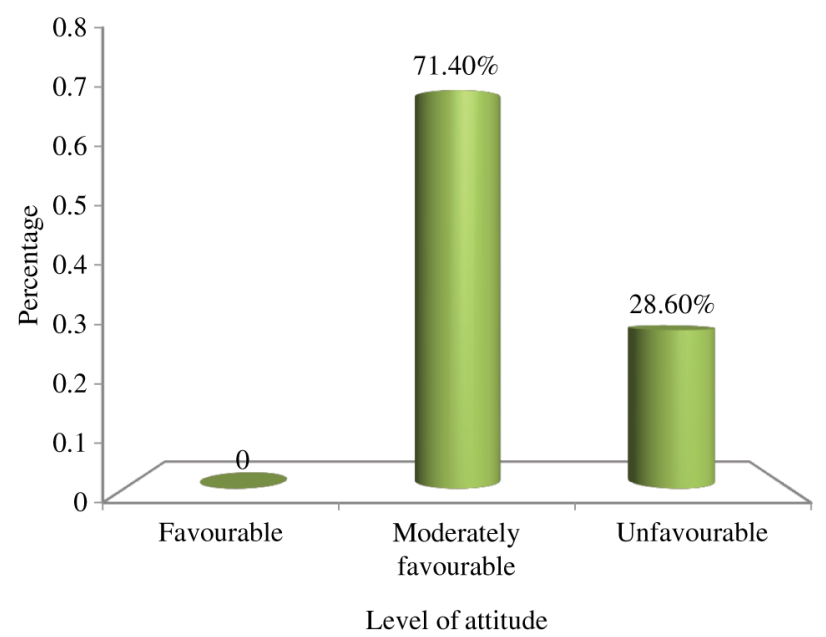

Figure 2: A bar graph showing percentage distribution level of attitude regarding reproductive and sexual health among teachers.

\section{Range, mean, median and SD of attitude score regarding reproductive and sexual health among teachers}

The data presented in Table 3 depicts the range, mean, median and the SD of the attitude score regarding reproductive and sexual health among teachers. The mean of the attitude score was 93.97 with a median of 93.50 and the SD was 8.33 .

Table 3: Range, mean, median and SD of attitude score regarding reproductive and sexual health among teachers.

\begin{tabular}{lccrr}
\hline & & & & $\mathbf{n}=\mathbf{7 0}$ \\
\hline Score & Range & Mean & Median & SD \\
\hline Attitude & $77-116$ & 93.97 & 93.50 & 8.33 \\
\hline
\end{tabular}

Maximum score - 150; minimum score - 30. SD, standard deviation.

\section{Correlation between knowledge and attitude scores regarding reproductive and sexual health among teachers}

Data showed the relationship between the knowledge scores and attitude scores among the teachers and it was found that there was mild negative $(-0.21)$ relationship between the knowledge scores and attitude scores which was found to be statistically non-significant $(p=0.862)$ at 0.05 level of significance. 


\section{Association of knowledge scores and attitude scores regarding reproductive and sexual health among teachers with selected variables}

The data showed that the knowledge scores and attitude scores were independent of age, gender, education status, experience in teaching, teaching about reproductive and sexual health education, years of experience in teaching about reproductive and sexual health education, specific training, whether teachers feel it is necessary to teach about reproductive and sexual health education, how often they teach about reproductive and sexual health education, strategies used, comfort level and barriers faced by the teachers.

\section{Discussion}

In the current study, the majority (62.9\%) of the teachers had an average level of knowledge regarding reproductive and sexual health, these results were little different to the study done by Westwood and Mullan where the knowledge of all teachers was generally good, with $84 \%$ attaining 20 and above out of a total of 28 on the knowledge score [11]. The findings of the present study were noted to be similar to the findings of another study done in Enugu by Aniebue where $69(23.0 \%)$ had adequate knowledge of sex education [12].

In the present study, the majority $(71.4 \%)$ of the teachers had a moderately favorable attitude towards reproductive and sexual health. This finding was contradictory to the study conducted by Fentahun et al. [13] where the majority of teachers $(75 \%)$ were against the introduction of sex education. Whereas findings from the study conducted by Onwuezobe et al. [14] supported the results of the present study where the majority (67.6\%) of the teachers believed that sex education prevents unwanted pregnancy, $43.6 \%$ of the teachers believed that it prevented STDs/HIV, and 13\% found sex education was beneficial for family planning. The present study's finding was in concordance to the study conducted by Orji et al. [15] where study findings revealed that $90 \%$ of the teachers supported the introduction of sex education into the school curriculum.

The findings of the present study revealed that there was no association between the attitude of teachers with age, education status and teachers' comfort level in teaching about reproductive and sexual health education as the computed $\mathrm{F}$ value was non-significant at 0.05 level of significance. The results of the present study were contradictory to the study conducted by Onwuezobe [14] in assessing the attitude of teachers to sex education where older teachers between the ages of 45 and 49 years (86.8\%), were more willing to teach sex education, higher education qualification had a significant effect on their attitude towards sex education.

\section{Conclusion}

The study was conducted to assess the knowledge and attitudes regarding reproductive and sexual health using a knowledge questionnaire and attitude scale among teachers of selected schools of the Ambala District, Haryana. The current study suggests that various training programs should be initiated by community health nurses along with a block education officer for teachers regarding reproductive and sexual health where teachers should be taught about all aspects of adolescence, puberty and the major issues related to adolescent health and all the common problems of adolescence. So that teachers can help with the common problems of adolescence and can prevent further complications.

The majority of the population use mass media to update their knowledge horizon. There is a great need to educate our population and make them aware by providing right knowledge about puberty and changes during puberty. Mass media can influence a population in both positive and negative ways, so by using it efficiently and adequately we can overcome the various myths and misconceptions related to changes during puberty like reproductive and sexual changes among adolescents.

Funding: No funding sources.

Conflict of interest: None declared.

Ethical approval: The study was approved by the Institutional Ethics Committee. 


\section{References}

[1] Subrata S. Textbook of pediatric nursing. 1st ed. New Delhi: Jaypee Brothers Medical Publication; 2017. p. 153-63.

[2] Sexual Development \& Puberty (Adolescence) - Sexual development - healthcommunities.com. [cited 2019]an 18]. Available from: http://www.healthcommunities.com/sexual-development/puberty.shtml.

[3] Adolescent Sexual and Reproductive Health/Save the Children. [cited 2019 Mar 24]. Available from: https://www.savethechildren.org/us/what-we-do/global-programs/health/adolescent-sexual-and-reproductive-health.

[4] Kumar Bhasin S, Aggarwal OP. Perceptions of teachers regarding sex education in national capital territory of Delhi. Indian ] Pediatr 1999;66(4):527-31.

[5] Nair MK, Leena ML, Paul MK, Pillai HV, Babu G, Russell PS, et al. Attitude of parents and teachers towards adolescent reproductive and sexual health education. Indian ] Pediatr 2012;79(S1):60-3.

[6] Fentahun N, Assefa T, Alemseged F, Ambaw F. Parents' perception, students' and teachers' attitude towards school sex education. Ethiop J Health Sci 2012;22(2):99-106.

[7] Tripathi N, Sekher TV. Youth in India ready for sex education? Emerging evidence from national surveys. In: Murthy AK, editor. PLoS One 2013;8(8):e71584.

[8] Orji EO, Esimai OA. Introduction of sex education into Nigerian schools: the parents', teachers' and students' perspectives. ] Obstet Cynaecol 2003;23(2):185-8.

[9] Kumar Bhasin S, Aggarwal OP. Perceptions of teachers regarding sex education in national capital territory of Delhi. Indian ] Pediatr 1999;66(4):527-31.

[10] Titiloye MA, Ajuwon A]. Knowledge and quality of adolescents reproductive health communication between parents and their adolescents children in Ibadan, Nigeria. J Public Health Africa 2017;8:688.

[11] Westwood ], Mullan B. Knowledge and attitudes of secondary school teachers regarding sexual health education in England. Sex Educ 2007:7(2):143-59.

[12] Aniebue PN. Knowledge and attitude of secondary school teachers in Enugu to school based sex education. Niger] Clin Pract 2007:10(4):304-8.

[13] Fentahun N, Assefa T, Alemseged F, Ambaw F. Parents' perception, students' and teachers' attitude towards school sex education. Ethiop J Health Sci 2012;22(2):99-106.

[14] Pakistan journal of medical sciences quarterly. Professional Medical Publications; 2001 [cited 2019 ]an 25]. Available from: https://www.pjms.com.pk/issues/octdec209/article/article11.html.

[15] Orji EO, Esimai OA. Introduction of sex education into Nigerian schools: the parents', teachers' and students' perspectives. ] Obstet Cynaecol 2003;23(2):185-8. 